\title{
Thermo-Mechanical Fatigue of Grey Cast Iron for Cylinder Heads - Effect of Niobium, Molybdenum and Solidification Time
}

\author{
Peter Skoglund ${ }^{1, a}$, Jessica Elfsberg $^{1, b^{*}}$, Nulifer Ipek $^{1, c}$, \\ Lucian Vasile Diaconu ${ }^{2, \mathrm{~d}}$, Mari Larsson ${ }^{3, \mathrm{e}}$, Pål Schmidt ${ }^{4, f}$ \\ ${ }^{1}$ Scania CV AB, S-151 87 Södertälje, Sweden \\ 2Jönköping University, Box 1026, SE-551 11 Jönköping, Sweden \\ ${ }^{3}$ Volvo Group Trucks Operations, S-541 87 Skövde, Sweden \\ ${ }^{4}$ Volvo Group Trucks Technology, Gropegårdsgatan, SE-405 08 Göteborg Sweden \\ apeter.skoglund@scania.com, ${ }^{b}$ jessica.elfsberg@scania.com, ${ }^{c}$ nulifer.ipek@scania.com, \\ dLucian-Vasile.Diaconu@ju.se, e mari.larsson@volvo.com, pal.schmidt@volvo.com
}

Keywords: grey cast iron, static mechanical properties, thermomechanical fatigue

\begin{abstract}
Grey iron alloyed with molybdenum and niobium in seven different compositions has been casted using three, in industrial components viable, solidification times which resulted in 21 different samples. The samples have been investigated with respect to microstructure, static properties and thermo-mechanical fatigue performance. It was found that the solidification time is very important for both the static and thermo-mechanical performance. If the solidification time is long the properties are controlled entirely by the large graphite flakes and there is no influence of the alloying elements. On the other hand if the solidification time can be kept short the need for alloying elements may be removed. For the shorter solidification times an influence from the matrix and thus the alloying elements can be seen. It was found that molybdenum enhances TMF-life while no such effect was found for niobium. Niobium, on the other hand, has a larger effect on static strength than molybdenum and also on the cyclic stress in the thermo-mechanical fatigue experiments.
\end{abstract}

\section{Introduction}

Demanding environmental legislation as well as requirements of decreasing fuel consumption and increased specific power for combustion engines inevitably lead to increased combustion pressure and temperature. A critical component in the engine is the cylinder head which is subjected to these higher fatigue loads. The enhanced high frequency and low amplitude pressure pulses increase the elastic high cycle fatigue (HCF) load that affects part of the cylinder head. The increased temperature leads to an amplified low frequency thermo-mechanical fatigue (TMF) load. For the cylinder head the TMF-load is critical as it affects the area between the valves. On heating it experiences high compressive stresses giving plastic deformation and when cooled down, tensile stresses develop initiating cracks and crack propagation in the subsequent cooling cycles. This damage process occurring close to the fire deck is often life limiting for the cylinder head. Further, in some cases the HCF-load is superimposed to the TMF-load which may lead to a large decrease in life, $[1-3]$.

Today, different lamellar graphite irons (LGI) or compacted graphite irons (CGI) are used for cylinder heads in heavy duty combustion engines. The CGI alloys have a considerable higher static strength and improved high cycle fatigue properties compared to LGI. However, the lower thermal conductivity of CGI gives higher thermally induced stresses for these type of alloys. Further, CGI also shows a higher E-modulus which also contributes to higher stresses for CGI relative to LGI in a strain controlled TMF-loading. As a consequence of the many factors influencing the TMF performance, CGI alloys may even be inferior to LGI alloys depending on the specific TMF-load conditions. The TMF-lives of some LGI and CGI alloys have been investigated and compared by 
different authors [1-6] showing the problem of choosing alloy. The increased complexity of casting CGI which implies higher costs compared to LGI in combination with the above discussions regarding a possible decrease in TMF-life, has triggered an interest in development of new LGI alloys with improved TMF properties. Among these, the effect of alloying elements has been investigated together with the influence of solidification time during the casting procedure. In this paper, seven different LGI compositions subjected to three different solidification times giving 21 samples have been investigated with respect to static properties and TMF-performance.

\section{Experimental}

Sample preparation. Seven different melts were prepared as indicated in Table 1. In order to investigate the effect of different solidification times, the melts were poured into staircases as shown in Figure 1. A total of 14 staircases were cast in seven heats. For every heat, the melt from one ladle was poured into one to three staircases using coated furan bonded quart sand molds. The pouring temperature varied between $1309^{\circ} \mathrm{C}$ and $1331^{\circ} \mathrm{C}$. Each ladle had its specific chemistry originating from the same base melt. The base melt was prepared in a medium frequency induction furnace from $1200 \mathrm{~kg}$ solid cast iron, $100 \mathrm{~kg}$ steel and $2700 \mathrm{~kg}$ liquid cupola melted cast iron. The inoculant $\left(0.2 \mathrm{wt} \%\right.$ Superseed $\left.{ }^{\circledR} 75\right)$, niobium and tin adjustments were added to the ladle during filling, while the molybdenum content was adjusted for in the furnace. A small amount of graphite was added to the furnace to adjust for carbon losses between the heats. The chemical analysis from each ladle are shown in Table 1.

Table 1. Chemical analysis (in weight $\%$ ) on coins from remelted samples of the staircases.

\begin{tabular}{|c|l|l|l|l|l|l|l|l|l|l|l|}
\hline Staircase & $\mathbf{C}$ & $\mathbf{S i}$ & $\mathbf{M n}$ & $\mathbf{P}$ & $\mathbf{S}$ & $\mathbf{C r}$ & $\mathbf{N i}$ & $\mathbf{M o}$ & $\mathbf{C u}$ & $\mathbf{S n}$ & $\mathbf{N b}$ \\
\hline $\mathbf{2}$ & 3.26 & 1.85 & 0.51 & 0.03 & 0.07 & 0.13 & 0.06 & 0.04 & 0.79 & 0.03 & 0.01 \\
\hline $\mathbf{4}$ & 3.23 & 1.84 & 0.52 & 0.03 & 0.07 & 0.13 & 0.06 & 0.04 & 0.80 & 0.03 & 0.14 \\
\hline $\mathbf{6}$ & 3.25 & 1.83 & 0.52 & 0.03 & 0.07 & 0.13 & 0.06 & 0.14 & 0.80 & 0.03 & 0.05 \\
\hline $\mathbf{8}$ & 3.25 & 1.86 & 0.53 & 0.03 & 0.07 & 0.13 & 0.06 & 0.14 & 0.79 & 0.03 & 0.11 \\
\hline $\mathbf{1 2}$ & 3.29 & 1.86 & 0.51 & 0.03 & 0.08 & 0.13 & 0.06 & 0.28 & 0.79 & 0.03 & 0.02 \\
\hline $\mathbf{1 3}$ & 3.27 & 1.88 & 0.51 & 0.03 & 0.07 & 0.13 & 0.06 & 0.28 & 0.79 & 0.07 & 0.01 \\
\hline $\mathbf{1 4}$ & 3.25 & 1.86 & 0.54 & 0.03 & 0.07 & 0.13 & 0.06 & 0.28 & 0.79 & 0.03 & 0.19 \\
\hline
\end{tabular}

Casting simulation. The casting process of the staircases was simulated using the commercial software MAGMAsoft ${ }^{\circledR}$ version 5.3.0. In Figure 1 the geometry of the staircase is shown. The thicknesses of the different sections of the staircase, designed to give different solidification times, were as follows: $12 \mathrm{~mm}$ for the thinnest part, $30 \mathrm{~mm}$ for the medium thick part, and $90 \mathrm{~mm}$ for the thickest part.

Mechanical experiments. The mechanical experiments were done using a commercial servohydraulic mechanical testing devices from MTS. The thermomechanical fatigue (TMF) tests were done with a MTS system equipped with an induction heating system. The TMF experiments were conducted in the out of phase configuration (i.e. compressive loads at maximum temperature) with a temperature varying between 100 and $500{ }^{\circ} \mathrm{C}$. The heating and cooling ramps were set to $2{ }^{\circ} \mathrm{C} / \mathrm{s}$ with a hold time of $25 \mathrm{~s}$ at the higher and lower temperatures. Further, the mechanical strain was set to 0 at the lower temperature and $-0.54 \%$ at the maximum temperature resembling a fully confined loading condition. Failure was defined as the cycle with a tensile stress of $90 \%$ compared to the cycle with maximum tensile stress.

From the central parts of each staircase, seen in Figure 1, four specimens were extracted, one for tensile testing and three for TMF-tests. The tensile test specimen had a gauge diameter and length of $7 \mathrm{~mm}$ and $25 \mathrm{~mm}$ respectively, while the TMF specimens had a gauge diameter and length of $7 \mathrm{~mm}$ 
and $12 \mathrm{~mm}$. The Brinell hardness values (HB 2.5/187.5) were measured using one indent only (due to size restrictions on the prepared specimens) on all specimens. The pearlite micro-Vickers hardness (HV0.1) was evaluated for samples subjected to the high solidification rate as the average of 8 indents.

Evaluation of microstructure. The microstructural features of the specimens were evaluated using LOM (Carl Zeiss with Axiovision) and SEM (Carl Zeiss) with EDX. Unetched samples as well as samples etched in picric acid and nitric acid were investigated.

\section{Results}

The simulation results gave solidification times between about 200 and $1400 \mathrm{~s}$ depending on the staircase thickness, see Figure 1 [7].

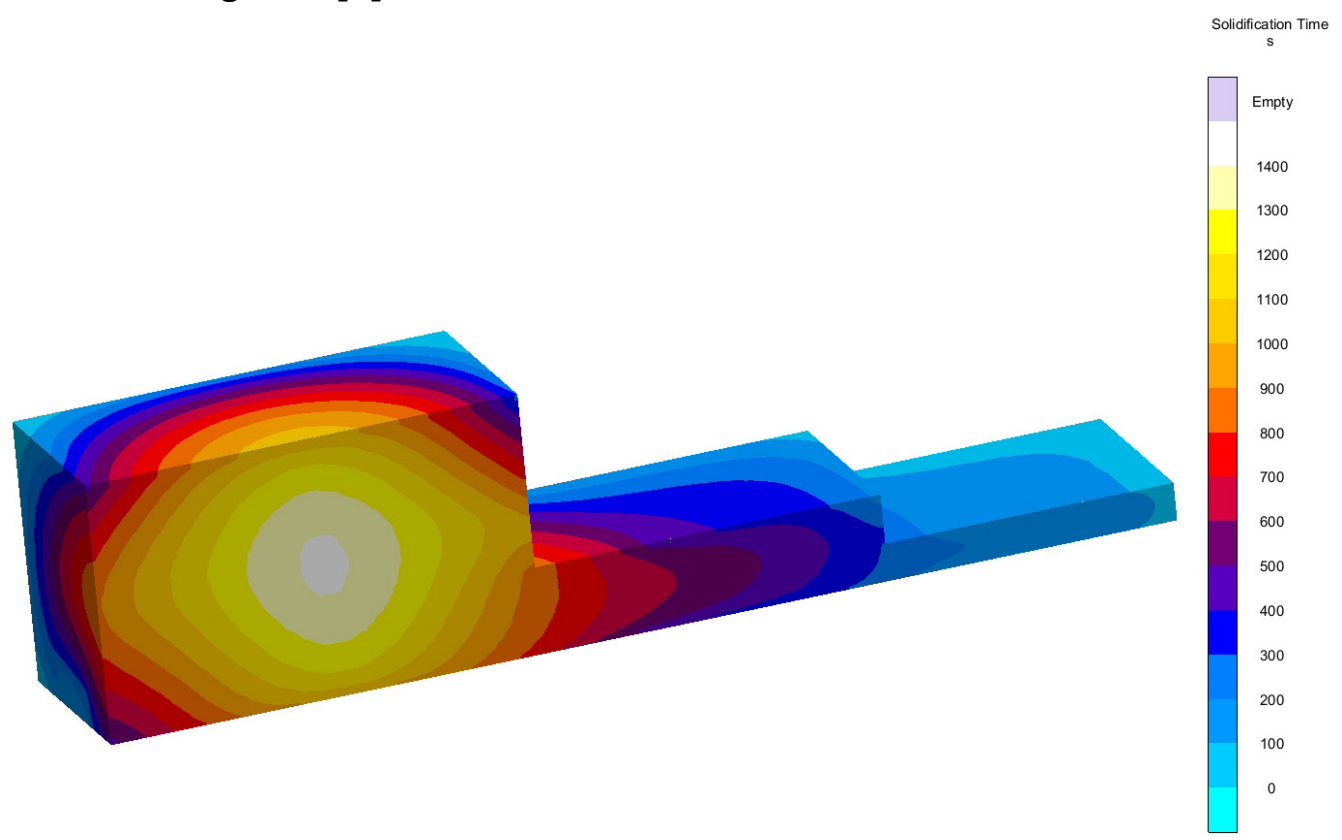

Figure 1. Simulation of filling of the staircase mould gives solidification times from about $200 \mathrm{~s}$ for the thin section to $500 \mathrm{~s}$ and $1400 \mathrm{~s}$ respectively for the medium and thick section respectively [7].

Table 2 summarizes the results from the static and thermomechanical fatigue testing as a function of solidification time and composition. From these results, it can be seen that the main influence on the static properties arises from the solidification time although the highly alloyed specimens also show improved mechanical strength compared to the un-alloyed specimen i.e. alloy 2 . The main improvement in static properties occurs between the long and the medium solidification times, while the increase in properties is lower going from medium to short solidification time. The results also indicate that niobium has a larger impact on the static properties compared to molybdenum although the small number of experiments makes it impossible to draw any definite conclusions. Further, the results also implies that the effect of solidification time is somewhat more pronounced for the un-alloyed specimen (i.e. specimen 2) compared to the other six alloyed specimens.

Regarding the thermo-mechanical properties, it was found (in agreement with the result for the static properties) that the fatigue life is highly dependent on the solidification time and that the TMF-life increases with roughly a factor of three, when the solidification time decreases from the longest to shortest, see Table 2 and Figure 2. 
Table 2. Mechanical properties from the static and the thermomechanical experiments.

\begin{tabular}{|c|c|c|c|c|c|c|c|}
\hline $\begin{array}{c}\text { Staircase } \\
\text { Composition } / \mathrm{wt} \%\end{array}$ & $\begin{array}{c}\text { E- } \\
\text { modulus } \\
/ \mathrm{GPa}\end{array}$ & $\begin{array}{c}\text { Yield } \\
\text { strength } \\
\mathrm{Rp}_{0.2} / \mathrm{MPa} \\
\end{array}$ & $\begin{array}{c}\text { Tensile } \\
\text { strength } \\
\mathrm{Rm} / \mathrm{MPa}\end{array}$ & $\begin{array}{c}\text { Fatigue } \\
\text { life } \\
\mathrm{N}_{\mathrm{f}}\end{array}$ & $\begin{array}{c}\text { Tensile stress } \\
\text { at } \mathrm{N}_{\mathrm{f}} / 2 \\
/ \mathrm{MPa}\end{array}$ & $\begin{array}{c}\text { Compressive } \\
\text { stress at } \mathrm{N}_{\mathrm{f}} / 2 \\
/ \mathrm{MPa}\end{array}$ & $\begin{array}{c}\text { Brinell } \\
\text { hardness / } \\
\text { HB2.5/187.5 }\end{array}$ \\
\hline \multicolumn{8}{|c|}{ Short solidification time $(300 \mathrm{~s})$} \\
\hline 2 (Unalloyed) & $125^{l}$ & 238 & 288 & 109 & 204 & -196 & $211\left(266^{3}\right)$ \\
\hline $4(\mathrm{Nb}=0.14 \%)$ & $120^{l}$ & 272 & 339 & 100 & 233 & -211 & $235\left(336^{3}\right)$ \\
\hline $6(\mathrm{Mo}=0.14 \%)$ & $113^{l}$ & 258 & 315 & 138 & 220 & -210 & $224\left(303^{3}\right)$ \\
\hline $8(\mathrm{Mo}=0.14 \% ; \mathrm{Nb}=0.11 \%)$ & $112^{l}$ & 249 & 304 & 135 & 226 & -212 & $228\left(275^{3}\right)$ \\
\hline $12(\mathrm{Mo}=0.28 \%)$ & $114^{l}$ & 252 & 315 & 162 & 216 & -230 & $225\left(270^{3}\right)$ \\
\hline $13(\mathrm{Mo}=0.28 \% ; \mathrm{Sn}=0.07 \%)$ & $116^{l}$ & 264 & 328 & 175 & 214 & -222 & $226\left(312^{3}\right)$ \\
\hline $14(\mathrm{Mo}=0.28 \% ; \mathrm{Nb}=0.19 \%)$ & $120^{l}$ & 274 & 339 & 184 & 233 & -233 & $230\left(297^{3}\right)$ \\
\hline \multicolumn{8}{|c|}{ Medium solidification time (500 s) } \\
\hline 2 (Unalloyed) & $98^{I}$ & 212 & 254 & 62 & 193 & -200 & 186 \\
\hline $4(\mathrm{Nb}=0.14 \%)$ & $115^{l}$ & 252 & 296 & 46 & 228 & -209 & 217 \\
\hline $6(\mathrm{Mo}=0.14 \%)$ & $116^{l}$ & 247 & 289 & 78 & 206 & -206 & 211 \\
\hline $8(\mathrm{Mo}=0.14 \% ; \mathrm{Nb}=0.11 \%)$ & $113^{l}$ & 248 & 291 & 69 & 214 & -214 & 216 \\
\hline $12(\mathrm{Mo}=0.28 \%)$ & $111^{l}$ & 249 & 297 & 96 & 219 & -226 & 219 \\
\hline $13(\mathrm{Mo}=0.28 \% ; \mathrm{Sn}=0.07 \%)$ & $111^{I}$ & 245 & 279 & 67 & 214 & -230 & 213 \\
\hline $14(\mathrm{Mo}=0.28 \% ; \mathrm{Nb}=0.19 \%)$ & $122^{l}$ & 268 & 329 & 77 & 236 & -229 & 226 \\
\hline \multicolumn{8}{|c|}{ Long solidification time (1400 s) } \\
\hline 2 (Unalloyed) & $91^{2}$ & 138 & 163 & 52 & 127 & -176 & 171 \\
\hline $4(\mathrm{Nb}=0.14 \%)$ & $104^{2}$ & 170 & 191 & 39 & 149 & -192 & 187 \\
\hline $6(\mathrm{Mo}=0.14 \%)$ & $99^{2}$ & 161 & 185 & 56 & 143 & -200 & 181 \\
\hline $8(\mathrm{Mo}=0.14 \% ; \mathrm{Nb}=0.11 \%)$ & $104^{2}$ & 168 & 190 & 45 & 150 & -208 & 187 \\
\hline $12(\mathrm{Mo}=0.28 \%)$ & $104^{2}$ & 155 & 171 & 25 & 145 & -209 & 176 \\
\hline $13(\mathrm{Mo}=0.28 \% ; \mathrm{Sn}=0.07 \%)$ & $101^{2}$ & 167 & 185 & 30 & 142 & -213 & 186 \\
\hline $14(\mathrm{Mo}=0.28 \% ; \mathrm{Nb}=0.19 \%)$ & $114^{2}$ & 201 & 227 & 78 & 172 & -221 & 191 \\
\hline
\end{tabular}

${ }^{1 .}$ Evaluated between 25 and $75 \mathrm{MPa}{ }^{2}{ }^{2}$ Evaluated between 0 and $40 \mathrm{MPa} .{ }^{3}$ Micro-Vickers hardness (HV0.1) measured on pearlitic areas as the average of 8 different indents.

In figure 2 the cyclic tensile stress at half-life $\left(\mathrm{N}_{\mathrm{f}} / 2\right)$ is shown versus the number of cycles to failure. As seen, the solidification time is very important also for the TMF performance. The experimental results group together depending on the cooling conditions. A long solidification time gave both short fatigue life and low cyclic stress value. Decreasing the solidification time from long to medium resulted in higher stress values for all samples (on average about 40-50\%) and most specimens also exhibited an increase in fatigue life. A further decrease in solidification time i.e. from medium to short increased the life for all samples at more or less constant stress values.

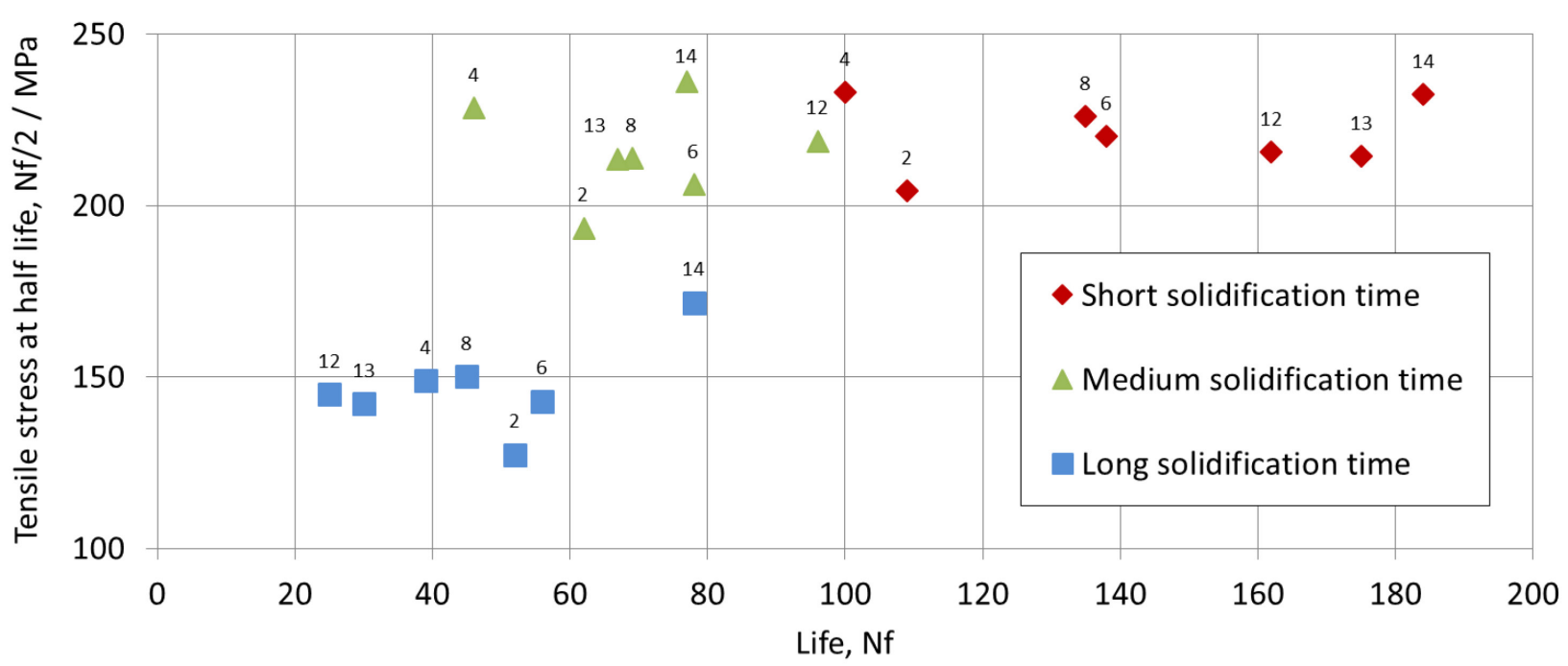

Figure 2. Tensile stress at half-life vs TMF cycles. The numbers indicate composition, see Table 1. 
It is also seen that for the short and to some extent medium solidification times, the alloyed specimens showed generally improved properties compared to the un-alloyed specimen labelled 2 . Although, for alloy number 4 , with only $\mathrm{Nb}$ added to the original chemistry, only the stress values are improved with no positive effect on the TMF-life. Further, for the longest solidification time, the results implied that the effect of alloying gets diminished by the attained coarsening of the structure. It is also noted that molybdenum improved the TMF-life more than niobium, while niobium seemed to be more effective in increasing the stress values at half-life and also the static strength seen in Table 2. In an effort to quantify this, regression analyses were done showing the relative importance of the alloying elements $\mathrm{Nb}$ and Mo on the TMF-life $\left(\mathrm{N}_{\mathrm{f}}\right)$ and tensile strength $\left(\mathrm{R}_{\mathrm{m}}\right)$ respectively and the results are shown in Equation 1 and 2.

$$
\mathrm{N}_{\mathrm{f}}=91+285 \cdot \mathrm{Mo}+35 \cdot \mathrm{Nb} \text {. }
$$

From the analyses it is seen that the molybdenum content gives a considerably higher impact on the TMF life compared to niobium. On the other hand, niobium was found to be slightly more important than molybdenum when it comes to improving the tensile strength as seen in Equation 2.

$$
\mathrm{R}_{\mathrm{m}}=294+68 \cdot \mathrm{Mo}+163 \cdot \mathrm{Nb} \text {. }
$$

Both Equation 1 and 2 were derived for the specimens with the shortest solidification time. The trends for the static strength seem nevertheless to be similar for the specimens with the medium and long solidification times. The TMF-life seems however to be more dependent on solidification time and for the longest time, alloying seemed not to be important for the TMF-life, as indicated by Figure 2 and Table 2.

The microstructural analysis show that the graphite size is very large for the specimens manufactured with the longest solidification time as seen from Figure 3. The large graphite flakes control the mechanical properties of the alloys and the impact of other alloying elements are negligible. For the medium and short solidification time the graphite size is smaller and the properties of the matrix may influence the results of the mechanical experiments.

\begin{tabular}{|l|l|l|l|}
\hline & \multicolumn{3}{|c|}{ Graphite content [\%] } \\
\hline Composition & $\begin{array}{l}\text { Short } \\
\text { time }\end{array}$ & $\begin{array}{l}\text { Medium } \\
\text { time }\end{array}$ & $\begin{array}{l}\text { Long } \\
\text { time }\end{array}$ \\
\hline 2 & 10.6 & 9.8 & 12.1 \\
\hline 4 & 10.1 & 10.0 & 10.2 \\
\hline 6 & 8.0 & 9.4 & 8.8 \\
\hline 8 & 8.7 & 10.1 & 11.1 \\
\hline 12 & 9.7 & 10.1 & 11.4 \\
\hline 13 & 10.6 & 9.6 & 10.5 \\
\hline 14 & 9.9 & 10.6 & 10.5 \\
\hline
\end{tabular}
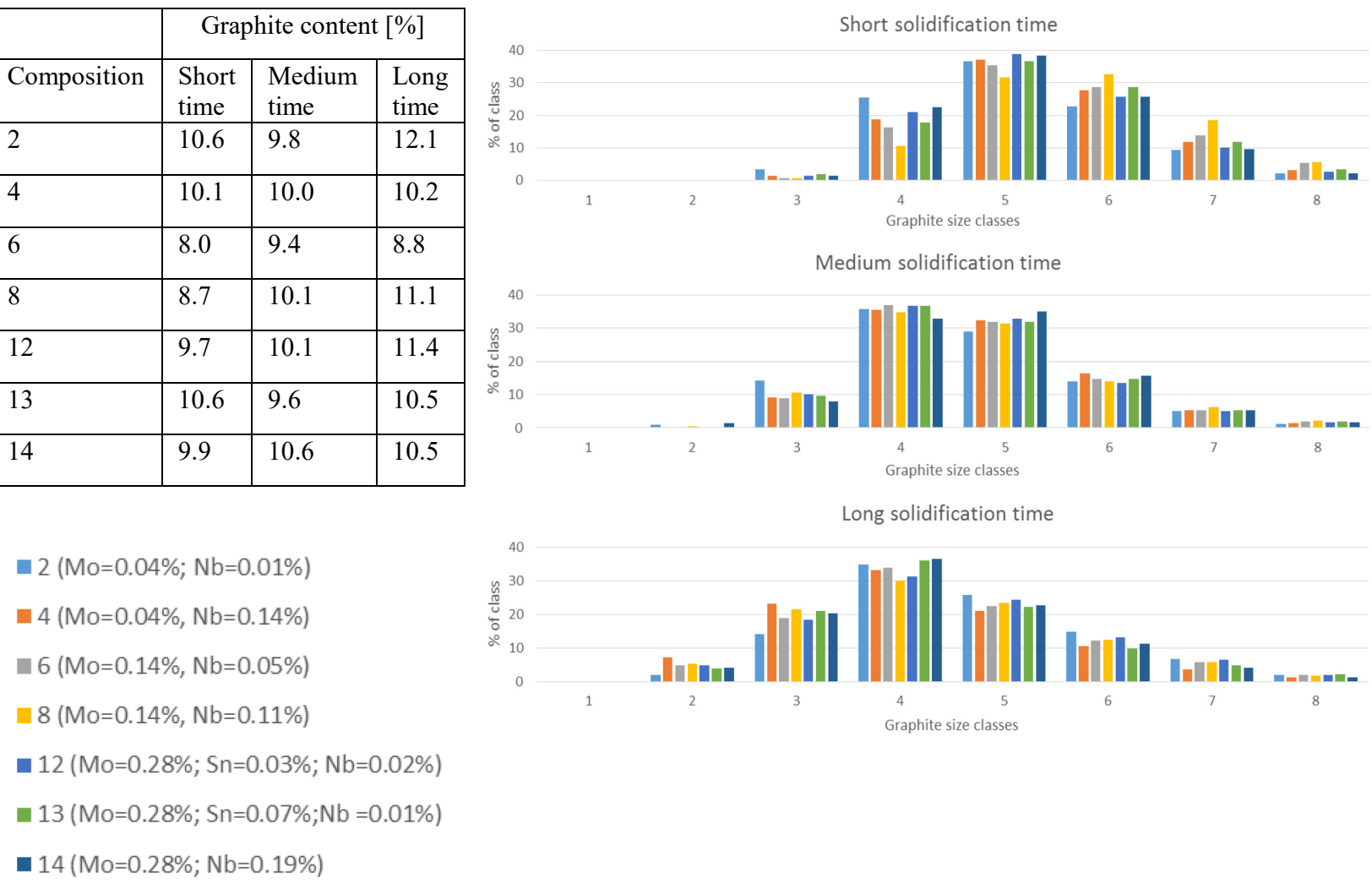

Figure 3. Graphite content and graphite size distributions for the different samples. 
In Figure 4 typical microstructures are shown for the compositions subjected to the short solidification time. It is difficult to make quantitative conclusions from the data, however some qualitative results can be extracted. First, all specimens are mainly pearlitic and it is noted that the dendritic structure shown in the first column, is refined for the alloyed specimens compared to the un-alloyed sample 2. Interesting is the bulky particles seen in alloy 4, 8 and 14 in the last column identified as niobium carbides that are not found in the non-niobium alloys labelled 2, 6, 12 and 13 .

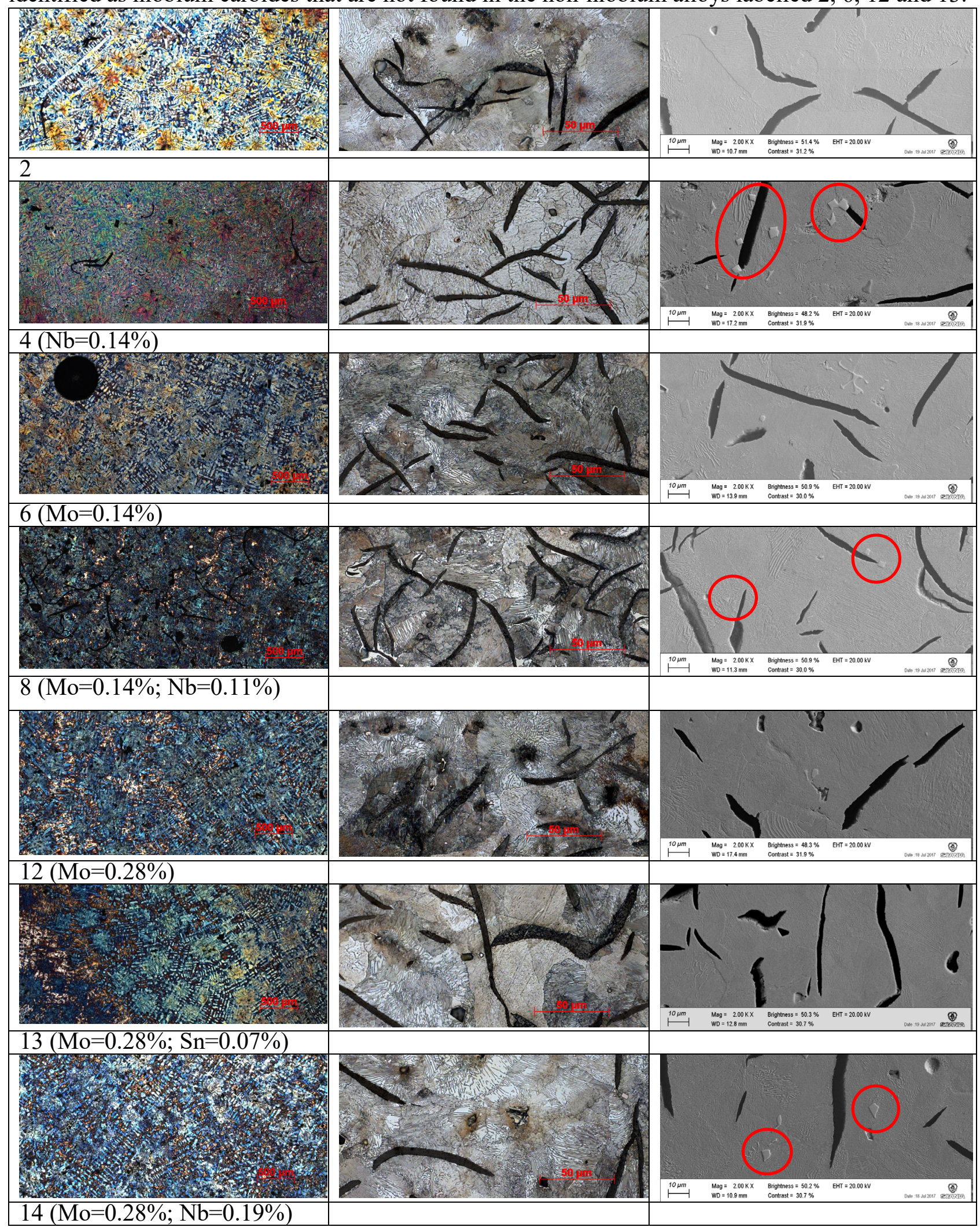

Figure 4. Typical microstructures for the compositions subjected to short solidification time. The red circles indicate bulky niobium carbides in specimen 4, 8 and 14 . 


\section{Discussion}

From the data presented above it is found that the solidification time is very important to not only the static properties but also for the TMF-performance. It is also emphasized that the investigated solidification times are rather typical for industrial castings, for example the short solidification time used in this investigation is close to what can be found at the top of a cylinder head. The importance of solidification time on the microstructure of grey irons is investigated by for example Jabbari and Behnam [8] showing that an increased cooling rate (i.e. short solidification time) is accompanied by a refinement of the dendritic structure as well as the pearlite lamellae thickness and as a consequence an increased Brinell hardness. An increased Brinell hardness with decreased solidification time for a given composition is also found in this investigation as seen from Table 2 . The microstructural analysis shows that the graphite particle size is very large for the specimens manufactured with the longest solidification time. The large graphite flakes control the mechanical properties of the alloys and the impact of the matrix and other alloying elements are negligible in this case. For the medium and short solidification times the graphite size is smaller and thus the matrix may have a larger impact on the mechanical properties. It is interesting to note that the static strength and cyclic stress increase more when going from long to medium solidification time than from medium to short. On the other hand the TMF-life increases more when going from medium to short solidification time compared to from long to medium time. The increase in strength and stress may be explained by the graphite size distribution as indicated by Figure 3. The static properties are controlled by the largest graphite flakes and thus a large increase in strength is found if the formation of large flakes are avoided. The cyclic stress values also increases with the removal of these flakes possibly partly also because the E-modulus of the specimens increases, see Table 2, and an increased stiffness gives a higher stress in a strain controlled TMF-experiment. The TMF-life is controlled by multiple micro-cracks initiated very early in the life and growing independently from each other from one graphite flake to another until they coalesce into a larger macro-crack close to failure $[1,2,4]$. Since the stress levels that drives the crack propagation are very similar for the medium and short solidification times, the increased TMF-life is attributed to either an increase of the total crack length or to an increased crack propagation resistance of the matrix when the solidification time goes from medium to short.

For the short, and to some extent the medium, solidification time adding Mo increases the fatigue life which most likely is due to changes of the crack propagation resistance in the matrix. For the short solidification time a relation between the austenite dendrite fraction and the number of TMF cycles to failure could be seen. The correlation factor between TMF-life and austenite fraction was in this case 0.82 , while the corresponding correlation factors for the medium and long solidification times were insignificant. A further analyses of the microstructure of the specimens cast with short solidification time shows that Mo and $\mathrm{Nb}$ additions to the original LGI seems to alter several details in the structure, unfortunately all of them are hard to quantify. The number and sizes of the eutectic cells as well as of the intercellular regions are different causing different segregation patterns. Furthermore, the pearlite lamellar distance alters but also the pearlite lamellas themselves. In some samples the lamellas are more irregular than in others. This difference may be the reason for the difference in pearlite hardness. Further, the Mo- and Nb-additions may cause carbide precipitation. The bulky rectangular shaped carbide particles are suggested to be connected to $\mathrm{Nb}$, as also found in the work by Wenbin with co-workers [9] when adding $0.29 \% \mathrm{Nb}$ to a hypoeutectic grey iron. For Mo-contents in excess of $0.95 \% \mathrm{Xu}$ et. al. [10] found Mo-carbides at eutectic cell boundaries in compositions in other respects similar to the ones investigated here. Sample 13 has a higher Sncontent than the other samples. The Sn-addition stabilizes the pearlite which explains the higher pearlite hardness as well as the structural difference between sample 12 and 13. In sample 12 some ferrite precipitations can be seen which is an expected consequence of the Mo-addition. In this respect, it is interesting to note that $\mathrm{Xu}$ [10] has shown that ausferrite (bainitic ferrite embedded in prior austenite) can be formed on direct casting by alloying with for example Mo. Niobium on the other hand is expected to stabilize and refine the pearlite structure [8]. Further, from the present 
investigation it is also seen that niobium increases static strength and cyclic stress, the higher stress values may be detrimental to the TMF-life since higher cyclic stress increases the crack growth rate. However, the HCF properties are likely to increase with the increasing strength. An interesting combination would then be to keep a short solidification time and add Mo to further enhance TMFlife and add $\mathrm{Nb}$ to improve the static strength and the HCF-life.

\section{Summary}

It is found that the solidification time of lamellar grey iron is very important not only to the static properties but also to the thermo-mechanical fatigue properties of the alloys. If the solidification time is too long the properties will be very poor regardless of alloying. On the other hand, if the solidification time can be kept short it may be possible to reduce the alloying while maintaining good mechanical properties and thermo-mechanical fatigue life. The poor properties of the long solidification time specimens are attributed to the large graphite cell size which in this case controls the mechanical properties. For the shorter solidification times an influence from the matrix and thus the alloying elements can be seen. It is found that molybdenum enhances TMF-life while no such effect is seen for niobium. Niobium on the other hand has a larger effect on static strength than molybdenum and also on the cyclic stress in the thermo-mechanical fatigue experiments.

\section{Acknowledgements}

The present study was conducted with financial support from Vinnova grant no 2013-03303.

\section{References}

[1] V. Norman, P. Skoglund, D. Leidermark and J. Moverare, The effect of superimposed highcycle fatigue on thermo-mechanical fatigue in cast iron, Int. J. of Fatigue, 88, (2016), 121-131.

[2] V. Norman, P. Skoglund, D. Leidermark and J. Moverare, Thermo-mechanical and superimposed high cycle fatigue interactions in compacted graphite iron, Int. J. of Fatigue, 80, (2015), 381-390.

[3] M. Metzger, B. Nieweg, C. Schweizer and T. Seifert, Lifetime prediction of cast iron materials under combined thermomechanical fatigue and high cycle fatigue loading using a mechanism-based model, Int. J. of Fatigue, 53, (2013), 58-66.

[4] V. Norman, P. Skoglund and J. Moverare, Damage evolution in compacted graphite iron during thermomechanical fatigue testing, Int. J. of Cast Metals Research, 29, Nos 1 \& 2, (2016), 25-32.

[5] T. Seifert, P. von Hartrott, K. Boss and P. Wynthein, Lifetime Assessment of Cylinder Heads for Efficient Heavy Duty Engines Part I: A Discussion on Thermomechanical and High-Cycle Fatigue as Well as Thermophysical Properties of Lamellar Graphite Cast Iron GJL250 and Vermicular Graphite Cast Iron GJV450, SAE Int. J. of Engines, 10, April 2017, 359-365.

[6] R. Hazime, T. Seifert, J. Kessens and F. Ju, Lifetime Assessment of Cylinder Heads for Efficient Heavy Duty Engines Part II: Component-Level Application of Advanced Models for Thermomechanical Fatigue Life Prediction of Lamellar Graphite Cast Iron GJL250 and Vermicular Graphite Cast Iron GJV450 Cylinder Heads, SAE Int. J. of Engines, 10, April 2017, 350-358.

[7] N. Oria, Master Thesis: Development of a Computer Model for Tensile Strength Prediction of Grey Cast Iron, Volvo report ER-709117, (2015).

[8] M. M. Jabbari Behnamn, P. Davami and N. Varahram, Effect of cooling rate on microstructure and mechanical properties of gray cast iron, Mat. Sci. and Eng. A 528, (2010), 583-588.

[9] Z. Wenbin, Z. Hongbo, Z. Dengke, Z. Hongxing, H. Qin and Z. Qijie, Niobium alloying effect in high carbon equivalent grey cast iron, China Foundry, 8, February 2011, 36-40.

[10] W. Xu, M. Ferry and Y. Yang, Influence of alloying elements on as-cast microstructure and strength of gray iron, Mat. Sci. and Eng. A 390, (2005), 326-333. 